\title{
Outdoor Collaborative and Creative Space Renewal in a Smart City
}

\author{
Rossella Maspoli ${ }^{1, a}$ \\ ${ }^{1}$ DAD - Department of Architecture and Design, Politecnico di Torino, v.le Mattioli 39, 10125 Torino, \\ Italy \\ a rossella.maspoli@polito.it
}

Keywords: Art, Public Outdoor Space, Urban Regeneration, Community, Smart Technologies.

\begin{abstract}
The paper analyzes the urban transformation and the development of criteria for the conception and design of outdoor urban space, in the smart city context. In the regeneration of peripheral historical and postindustrial neighborhoods, interactive storytelling and cultural mediation for collaborative placemaking of public sites can generate not only art and culture - in accordance with the enhancement of historical memory and to the rediscovery of local identity - but also opportunities for redevelopment.
\end{abstract}

\section{Introduction}

The paper analyzes the urban transformation and the development of criteria for the conception and design of outdoor urban space, in the smart city context.

In the regeneration of peripheral historical and postindustrial neighborhoods, interactive storytelling and cultural mediation for collaborative placemaking of public sites can generate not only art and culture - in accordance with the enhancement of historical memory and to the rediscovery of local identity - but also opportunities for redevelopment.

The research evaluates case studies and explores the potential of innovative micro-community aggregation through the social media interaction, the analysis of use and performance requirements for public space and the experimentation of from the bottom construction of new services and equipment through an interdisciplinary collaborative network. The network promises constituted by citizens, community facilitators, professional experts, young in training creative and local artisan entrepreneurs.

The collaborative placemaking focuses on the design and construction of eco-friendly and recycling equipment and on the sharing services for the use of marginal outdoor spaces and the re-use of abandoned spaces on the ground floor of buildings.

The plan of operations research is to establish a creative supply chain, from the development of a web platform for sharing spatial data and a 'map of the community' to the construction of hybrid places - real and digital - through processes of traditional handcrafts such as digital fabrication, to improve the quality of living, the leisure and the health.

\section{Smart City and outdoor public spaces quality}

A Smart City must offer varied social and economic opportunities and must have high quality public spaces.

The perception of an urban space can significantly affect the use that space, and the collective opinion that a community has of such space shape influences the wider community's image of urban spaces. We must treat all streets as open quality spaces for different users and the new public spaces must be for knowledge workers.

From a design perspective, new technologies provide ways of adding value to the architectural barriers and the outdoor spaces, they are producing fusion spaces [1], outdoor spaces in which digital electronic technology enables new and socially valuable combinations of people and activities. 
The local image, however, holds an important role in the territorial re-development, thus, turning the local governments in to a kind of image-maker [2]. The local governments' regeneration projects are carried out within a participatory and multi-sector view to increase the cultural level at the local context, to establish new media of interaction, to enhance the local attractiveness, economic and touristic.

Nowadays, the roles assigned to the peripheral neighborhoods are those of housing, leisure activities and provision of art facilities, but also - as Jeremy Rifkin pointed out [3] - the innovative and creative factory of the 'Third Industrial Revolution' can once again become compatible with a location inside the dense city, into a new functional mixture. This kind of a transformation appears to be the reformulation of the concepts of urbanity and urban way of life, meanings that vary according to ethnic origin, social status, age and digital capacities and that are indeed relevant in the sense of different demands of public spaces.

The research is addressing issues of outdoor public space in different perspectives: the role of digital mapping tools for the reconstruction of the meaning and memory of urban communities, the ability of local communities to define their own active demand of public space use, the potential of placemaking for collaborative design of collective space and construction of equipments, the improvement of the quality performances and the sustainability management, the influence of interaction design in a virtual / physical outdoor space [4].

\section{Local identity construction}

In human geography, place can be distinguished from outdoor space in terms of its identity which is assigned by its users, it is a kind of elements upon the value of which are concentrated [5]. The meaning of a place is construed through a reciprocal and dynamic process between the individual being and the collective mind of the users.

The focus is on the potential role of social networks communities and of traditional local community for the re-appropriation of the sense of place, the development of shared activities in the public space re-use and, therefore, the active interest in its conservation and management, defending it from vandalism. The shared memories can take on a strategic meaning to conservation / transformation projects with a view to place branding and strengthening.

A significant case study is the project for 'micro urban squares' of self-construction in public spaces - subtracted to the parking of cars - from the street community in the district S. Vitale with the 'Cultural Association Centotrecento' and the city of Bologna.

The 'micro square' is an architectural and urban design solution to solve problems of living in the suburbs, it is temporary equipped - in hot weather - in order to know and spend time together with the neighbors, taking care of the space and suggesting improvements. Regulations for use of 'micro neighborhood squares' has been agreed between the Association and the Municipality.

The goal is to create a support for people's daily life, to build together public spaces and the community that uses them, in reference to the model of the local action of the 'Transition Network Movement'. The Movement is one manifestation of the idea that local action can 'change the world', one attempt to create a supportive and healthy context. Nowdays, it is an experiment present in thousands of communities in 40 countries.

\section{Community mapping projects}

Community mapping projects [6] can be a important tool to foster partnerships and collaborative decision making skills. This type of projects can be used to develop important links between citizens, community agencies and local government. The crowdsourcing for social activism and the public accountability permits to develops open-source software for information collection, visualization, and interactive local mapping. For example, the MEIPI collaborative space allows a community of users to share information and mapping a local context, for collaborative dynamics and creative actions. 
In the community maps project in 'Barriera di Milano', Turin, the first expected result is a re-construction of the community-identity, in account of the social, architectural and economic changes.

The second expected result is the verification of methodologies for the promotion of the local districit - this considers the recognition of the identity up to its representation as emotional landscape comprehensible also for people who are outside of this community that has produced these values.

In changing and multicultural local society, the community is under construction, the social groups are fragmented and have low inter-communication. The problem is to identify the community that will be able to recognise the place, to map, to explain a significant demand for public space and collective services, for encourage creative and sustainable transformation processes.

From this point of view, a meaningful approach is the 'communicative turn' described by Patsy Healey [7], considering Habermas's communicative rationality and practical reasoning. Habermas asserts that the intersubjective practical reasoning draws on the store of knowledge and understanding of technique, morality and aesthetics. In this way, the intersubjective practices may involve telling stories as well as analysis, in the end, the results is 'acting in the world'. Further, the reasoning employed can include varying and cultural specific traditions of aesthetic experience and 'planning is a way of acting we can choose, after debate'. In according to this conception, the community maps project was proposed as a preparatory, dialogic and creative process in three phases.

In the first phase, the community map is introduced by the construction of psycho-geographic and creative maps by individuals inhabitants, about 200, belonging to the same micro-communities, through the project 'Create your map of Barriera' by the artist Nella Caffaratti.

The second phase of the mapping project has tried to restore the reading of the main spatial elements and spatial uses of certain micro-communities: ancient women who live in the district for a long time, young people with different backgrounds and ethnicity, artists and creative professionals who attend a public local .... The mapping process is promoted through the Facebook network to involve the informal community, people who can share stories or experiences.

With regard to the third phase project, several cartographic models are considered. Starting with the approach of imageability of the cognitive maps of Kevin Lynch and the experiences of "parish map' of Common Ground - from reproductive realism to a consciously factual and aesthetics map we have analyzed a series of digital design methodologies for participation, social inclusion and innovation: the sensorial maps - Christian Nold's Biomapping, Denis Wood's emotional infrastructure portrayals, online platform Drawing Emotions - which introduces an interactive design methodology to insert and manage each selected and geo-referenced element of public and private open and green spaces.

\section{Leisure and work in outdoor urban spaces}

The transformation will be in favour of public spaces if people will be attracted to new and reprogrammed functions, and to the reformulation of physical urban space while considering the interaction of leisure and work time activities [8].

For example, the aim of the project 'Smart Work@IJburg' of the City of Amsterdam is to realize a new kind of co-work centers. The IJburg district citizens will be faced with the possibilities to work more flexibly and especially near their homes and the Amsterdam Smart City will launch an awareness campaign to convince people to work in a smarter manner. The smart work stations are pre-equipped (digital system, telepresence control ...) enabling the technological quality currently available. They intend - like Smart Work Centers of 'Creative Wallonia' in Belgium - to meet workers in terms of flexibility, mobility and productivity. The innovative co-work stations may be hosted in spaces with different activities (community centers, libraries, commercial premises...) in public-private partnership, open to employees and professionals for an unlimited time, without having to face the necessity of often nerve-racking and time-consuming commuter traffic.

The evolution of co-working concept draws attention to the contiguous internal - external spaces, moving the digital office functions even in the open space, when the weather permits. The perspective 
is to extend the flow pattern of Moebius House by Van Berkel - the interlocking Moebius lines remind the formal organisation of the spaces and of the condensation of differentiating activities - in social inclusion spaces, where people feel welcome as they walk in from the street.

The hypothesis is to relate the participatory planning to make place - ie to improve the quality of marginal and unused spaces - with the promotion of street or neighborhood community, of creative activities to produce eco-friendly equipment for the collective space - tailored to the inhabitants - as well as local branding objects for fair trade in favour to the community, combining artistic creation and social commitment.

The main objectives are to organize field training and to support social youth work - in an urban area affected by the economic crisis - making local brand and signing the outdoor spaces (Fig. 1).

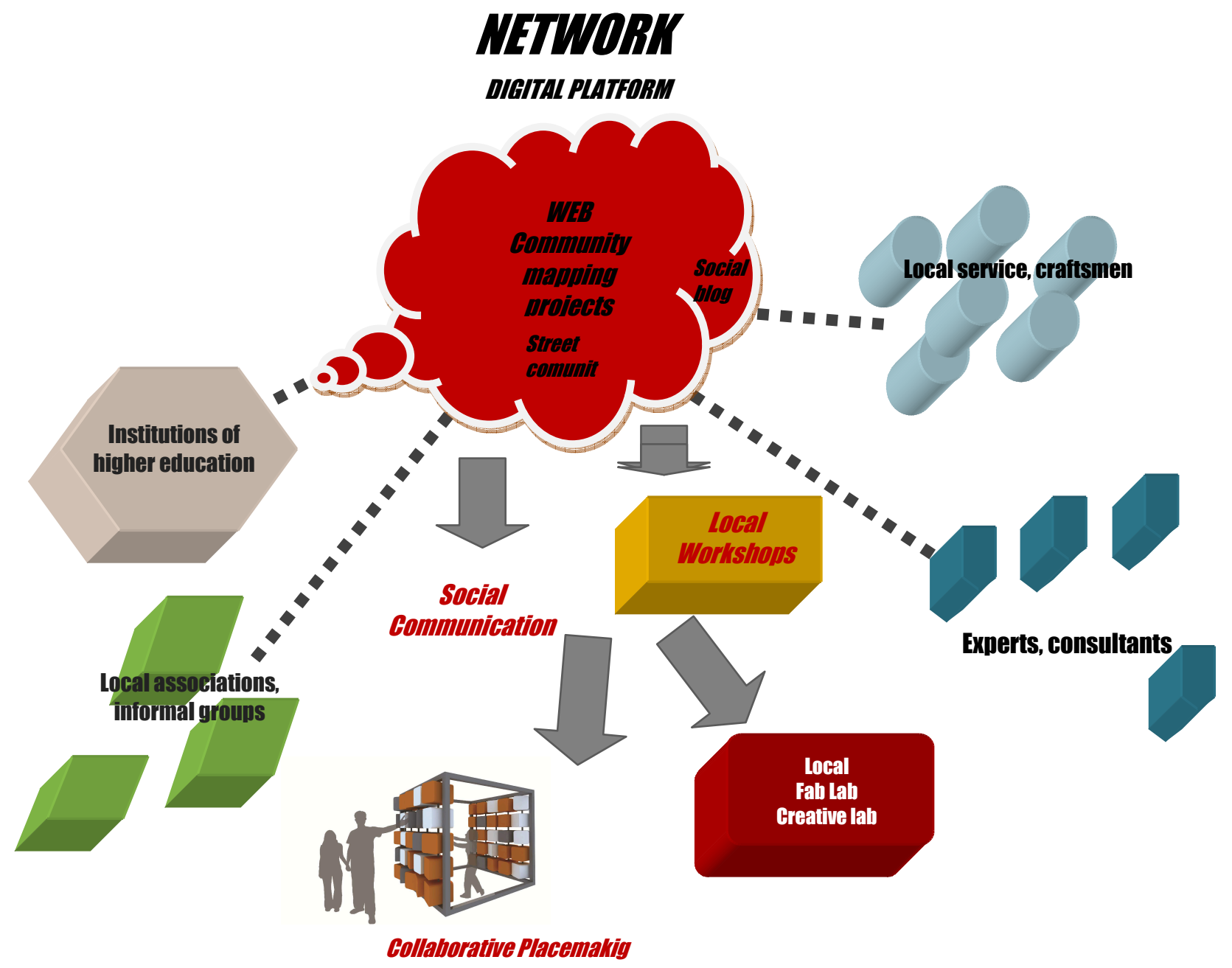

Fig. 1 Community mapping and placemaking project. Network and summary diagram.

\section{Polysemous concepts of public place}

A essential framework for evaluating real/virtual spaces is the respect to the polysemous concept of public sphere and the role will be the re-construction of public space meaning. Digital mediated communications generate an interactive process through space, the focus is on the changing of social places, where these communications are developing. 
Multi-user and hybrids environments, in which users can become participating actors, are examples of the socio-technical structure. The decentralized structure of virtual spaces suggests any re-conceptualization in the local social relations.

In this sense, virtual communities take the definition of public sphere on their non-commercial context, picturing not only a kind of interaction which can be called as text-based socialization, but encouraging new forms of perception, apperception and cognition.

In the project, the real and virtual contiguity between interior and exterior spaces - recognized and rebuild as places of identity - it is essential.

Hybrid space is a concept for integrating the real city and the parallel virtual city. Its design is based on an integration of information and architectural spaces, the implications of new practices require an empirical requirements study, informing the social design.

The space vision is - for individuals as well as communities - that the environment around us becomes an interface to information which can be (re)presented in many different forms. The innovative relationship is between the affordances provided by real, physical objects in the architectural space and digital information objects in the virtual information space, such as internet access points, interactive info-point, detection sensors, urban screens, co-working stations, stations for digital fabrication.

The project is also intended to experiment the ways in which report the virtualization physically ie the coverage of wifi and digital information objects - in the urban space, in order to increase the involvement of the people and to limit the digital divide.

\section{Collaborative place-making}

The key concept is the public renewal in oldest and suburban areas as a community-building asset, decreasing inequity in the distribution of usable space, creating new available space and increasing public outspaces quality. This approach requires to reinforce and encourage the micro-local communities to facilitate citizen-local government relations and to promote participatory, integrated and interactive public spaces design.

In the aim to re-designing to improve health and wellbeing, the 'Knee High Design Challenge' in England, thinks about how the design of public space can increase opportunities for play and leisure, at the neighborhood scale. The design can shape the physical, intellectual and emotional development of children and conversely, how children's play can inspire designers' ideas.

Active participation in the local urban design involves both the perspective of health and urban quality than that of the enhancement of the local cultures.

In a urban park project in Copenhagen, Norrebro district, called Superkilen, more than 50 different national communities have participated in the urban design, they were asked to nominate specific city objects such as benches, bins, trees, playgrounds, manhole covers, and signage from Country of origin. The objects were then either produced in a 1:1 copy or purchased and transported to the site. Realdania, a philanthropic business, financed the park's design and construction. A space decisional process like Superkilen could have been opened up to more people in the area and the selection of the park design made in public meetings, in particular, it highlights the difficulties in the maintenance and management of space.

Another urban park - result, instead, of consultation between the public and citizens - is Park Fiction, in Hamburg, is a collective project by active residents from St. Pauli. It's a green and small oasis in the middle of the town with a view of the harbour dockyard, an excellent example of how artists and activists can work with an informed local population to develop an exciting and innovative city space. The neighbourhood park has been designed by the residents (2005), it is located in a significant place, that the city's government wanted to sell to private investors.

In the contemporary urban regeneration, it is noted that [9] particular places orchestrate social behavior by providing scripts for encounters and assemblies. The built environment shapes individual's actions and identities by reinforcing relatively stable cues about correct and social 
behaviors. Public outdoor space uses physical and visual guides to produce desired behaviours: this perspective highlights the potential of shared design in marginal outdoor spaces.

In the pregress Barriera's research experience [10], sites become usable places even if they are only slightly equipped.

Some spatial characterizations to inhabit the outdoor public space have emerged from the participatory research: freely positioned seating, apparatus available for adult games as a way of bring different ethnic groups together, open booking lending service, symbolic objects and colours of different communities.

A first result in the listening and portrayal process concerns how to design spaces for inclusion, the metaphor of living is extended to outdoor spaces to home and how they can become welcoming and vehicles for memories, in a relational dimension. The square, the road, the garden and barriers can become emotional spaces and spaces of fusion, conditions of wellbeing.

The spatial and landscape context plays a decisive role. In this view the experimental asset is - such as in the "Workshop Performance in Art and the city. Architecture of outdoor public places in the Smart Cities', Turin, 2012, Valentino Castle - to hybridize, to interact with the place and to create a social interaction.

\section{Potential of placemaking actions}

In marginal public spaces, creating fusions of work space and social space - ie lounges with wireless connectivity, and quiet corners to work as well as areas for socializing - reduces isolation and increases opportunities for social exchange.

The place is changeable, the joint expression of multiple memberships puts into question the relationship between values, beliefs, imagination and materiality of signs, objects, buildings in outdoor public spaces.

Under this condition, the program of creative workshops promises to take advantage of the multi-cultural and professionalism in the neighborhood, according to the principles of self-production and technological hybridization, responding to the demand for personalized and low-cost spaces and objects. At the same time, labs can stimulate local and recognizable brand and a social merchandising, using social communication, product design, packaging, pricing, and multimedia display.

The laboratories of creative activities - located in abandoned sites in the neighborhood - can be initiated and managed through a local network, which involves young people in training, citizens, community facilitators, professional experts (artist, designer, architect, sociologist), young in training creative and local artisan entrepreneurs.

The legal-administrative form is a public-private partnership: granting use of public space and opening permit of private sites and rooms, that overlook the outdoor areas subject to intervention and can be used - with minimal costs of safety measures - for temporary.

The next phase of the research project is, therefore, aimed to test the collective design approach through the establishment of creative laboratories in partnership, available to local residents particularly young NEET (Not in Education, Employment or Training) people -, students and interdisciplinary experts.

According to the 'fab-labs' approach, the structures don't have to compete with mass production and its associated economies of scale, they have to show the potential to empower individuals to create smart devices or spaces for themselves or for the local communities.

Among the references, 'Waag Society', in Amsterdam, is considered. It is an interdisciplinary non-profit media lab researching, developing new technology, art and culture. The mission is to work in interdisciplinary teams to provide meaning and give direction to the role of technology in society and to contribute in interdisciplinary community building. In co-operation with end-users it develops technology that enables people to connect, product and share. The traditional techniques and the new 
technologies - such as GPS, fab-labs, cradle to cradle (regenerative design) - are to be explored and made usable, in relation to new forms of gaming, participation and distributed cooperation.

\section{Conclusion. A prospective in social smart city}

The public space design can play a key role in improving the lives in suburbs: outdoor play, leisure, co-work places become essential to healthy human lives, social connectivity, problem solving. Examples of the creative connection between modern technology and craft local traditions are emerging. The implications are huge, the practice of open and collaborative design is concerned both the objects that are made, both the relationships between producers - users. In the 90s, Ugo La Pietra wrote: 'To live or survive in the city? I always thought that a human being ensures its survival through the modification of the environment in which he lives and works, not only that, but I always believed that living a place means above all to be able to possess the mental point of view!' [11]. The focus is on the ability to collaborate in a shared way, on the process of making space. All local makers take responsibilities in the process of creating, for the elements used and for the social and environmental results.

In the Barriera's project, the results of the 'community mapping project' have allowed to preselect marginal and minor sites on which to develop the design process and define the equipment to be produced in the self-construction workshops.

The project responds to residents' needs to design and customize their outdoor living environment. In collaborative place-making, public space is conceived as a series of 'rooms to live,' the street of micro-communities become proactive to deal with a difficult urban situation. In reference to other European experiences, the activation of workshops will help to increase social inclusion and participation - planning together for a shared goal - and the communities will be able to translate their wishes into physical elements.

The project should not only lead to a new paradigm of construction, but also the open design principle renews the interaction between users, experts and trainees, is aimed at start-up of creative activities that have an economic return.

In urban critical neighborhoods, increasing a collaborative production chain of participative design is part of current European Community actions, according to a social and ethic prospective.

\section{Acknowledgements}

This work was financially supported in 2010 - 2013 by Archi.Pla.Architecture and places: local landscape valorisation between identity development and promotion. From «parish maps» to «territorial brands». Piedmont Region.

\section{References}

[1] W. Mitchell, E-topia: Information and Communication Technologies and the Transformation of Urban Life, The Network Society From Knowledge to Policy, Johns Hopkins Center for Transatlantic Relations, Washington (2005)

[2] A. Greenhill, G. Fletcher: The Social Construction of Electronic Space. Information on http://www.spaceless.com/papers/12.htm (2003)

[3] J. Rifkin: The Third Industrial Revolution; How Lateral Power is Transforming Energy, the Economy, and the World, Palgrave Macmillan, New York (2011)

[4] R. Maspoli: Technologies for urban microlandscapes in the smart city, in: R. Maspoli, M. Saccomandi: Art, architetture, landscape, Alinea, Firenze (2012)

[5] T. Hall: Urban Geography, Routledge, New York (1998) 
[6] Spatial Information design Lab, Community Mapping Project: A Guidebook for Neighborhood Associations and CDCs, Broodmor (2006)

[7] P. Healey: Planning through Debate: The Communicative Turn, in: Planning Theory, The Town Planning Review, vol. 63, n. 2. Liverpool University Press, Liverpool (1992)

[8] M. Kohn: Radical Space. Building the House of the People, Cornell University Press, Ithaca, NY (2003)

[9] Y. Tuan: Space and Place: the Perspective of Experience, University of Minnesota Press, Minneapolis (1977)

[10] R. Maspoli: Community maps. Recognition of the heritage and local enhancement, in: Architecture and places. Cultural design and site's memory, edited by M. Trisciuoglio, M. Barosio, M. Ramello Celid, Torino (2014)

[11] U. La Pietra: Abitare la città, Allemandi \& C, Torino (2011) 